\title{
Descriptor of self-determination based on cognitive models
}

\author{
Mikhail Rozin ${ }^{1 *}$, Valeriy Svechkarev ${ }^{1}$ and Zhanna Tumakova ${ }^{1}$ \\ ${ }^{1}$ Southern Federal University, Russia
}

\begin{abstract}
There is interest of scientists of various applied fields of science in the possibilities of research within the framework of the concept of self-determination. It is suggested to concentrate on the general semantic content of self-determination, which is inherent in it regardless of the applied focus of research. It is shown that self-determination is manifested as a system property of the sociocultural system activity as a whole. Furthermore, the level of self-determination of the sociocultural system and the potential for development in the target direction are directly proportional to the degree of semantic and causal integration of its elements. It has been identified that a set of factors united by cause-andeffect relationships in an oriented named signed graph reflects both the integration causation and the logical-semantic target organization of the sociocultural system. Thus, based on the figurative approximation of the essence of the studied system property of self-determination and its visual metaphorical representation in the form of a cognitive model, we get the opportunity to study the self-determination descriptor. Analysis of the causal integration of the elements of the sociocultural system or process using cognitive models makes it quite simple to determine the level of selfdetermination of a system not only at a qualitative but also at a quantitative level.
\end{abstract}

\section{Introduction}

Currently, domestic and foreign scientific literature presents a significant amount of theoretical research and many practice-oriented results related to the problem of selfdetermination and concerned with various fields of knowledge.

First of all, such works include the self-determination theory (SDT) in the field of psychology by American authors Edward L. Deci and Richard M. Ryan [1]. The authors give the following definition: "Self-determination theory aims to outline the factors that support the innate human potential for growth, integration and well-being and to study processes and conditions that contribute to the healthy development and effective functioning of individuals, groups and communities" [2]. In the work of Dergacheva O.E., there is a detailed analysis of the fundamental sources of the theory, and the following is postulated as a definition of self-determination: "Self-determination is the ability to choose and have a choice, in contrast to reinforced reactions, drive reductions and actions under the influence of other forces, which can also be considered as determinants of human

\footnotetext{
* Corresponding author: mdrozin@sfedu.ru
} 
behaviour" [3]. Self-determination (also defined by the "autonomy" descriptor) is based on intrinsically motivated activity.

\subsection{Acculturation, Stereotypes and Agency}

The propositions of SDT developed in the field of psychology aroused the natural desire of scientists to test, use or develop its concepts in various fields of knowledge. In the field of pedagogy in [4], the development of self-determination in adolescence is associated with the prevention of addictive behaviour, and the development of spiritual fullness of personality is seen as a basis for the formation of self-determination. In the field of education, the ideas of self-determination are used in the theory of self-determination [5]. The descriptor of self-determination in it is "a psychological construct that determines an act of will performed by a person of his/her own free will". In this case, the self-determined behaviour is already determined by intrinsic motivation, conscious choice and decision. In an organizational context in the field of sociology and political science, selfdetermination is introduced in a number of works as "a construct describing such a type of determination of behaviour when the factors initiating and regulating it arise from within the personality" [6]. Such behaviour is an end in itself and not a means to achieve some other goal - external or imposed from the outside. An even more peculiar approach to the determination of the self-determination descriptor is formed in forensic science (see, for example, [7] and [8]). Here, it is suggested to define self-determination as a process of generating new socially dangerous acts prohibited by criminal law. It is noted that organized crime relies on self-determination as an internal source of its reproduction. The things studied are internal determinants that determine the form of self-determination of crime.

Thus, the interest of scientists from various applied fields of science is based on the fundamental possibilities of obtaining the desired new results within the framework of the concept of self-determination. In so doing, it is possible not only to more deeply comprehend the situation, but to obtain reasonable and reliable research results. However, there has been also observed a traditional tendency for such rapid expansions of the application fields of the theory, namely, "blurring" of the self-determination descriptor itself, multiplicity of definitions resulting from a variety of scientific concepts, methodological disunity in assessing the quality of the self-determination potential, the achieved quantitative level, etc.

\subsection{Perspectives on Supervision}

The generally accepted definitions of the term descriptor refer to the need to describe the main semantic content in terms of a certain field of knowledge. With such a formulation, the revealed variety of descriptions of the self-determination descriptor in various scientific studies seems natural. In search for answers to this problem, the authors suggest in this article to concentrate on the general semantic content of self-determination, which is inherent in it regardless of the applied focus of research.

The search for such a general semantic content implies an appropriate level of abstraction from applied detailed elaboration. Pitirim Sorokin formulated this problem as follows: "The essence of the logical-semantic method of cognition is to find a central principle (argument) that permeates all components, gives meaning and significance to each of them, and thus turns the chaos of non-integrated elements into the single cosmos" [9].

Following Pitirim Sorokin, first of all, we come to realization of the considered property as a system property, inherent in the sociocultural system, and then to the definition of the necessary and sufficient features of the sociocultural system: "The first thing is the presence 
of causal (functional) relation and the second is the presence of the logical-semantic relation of the system elements (agents of actions and counter-actions), then there is empirical inclusion of the meanings of the system into sociocultural reality " [9]. So, within the framework of the sociocultural system, self-determination certainly gives a single meaning to the elements, agents, and components integrated into it. Moreover, with causal (functional) and logical-semantic (structural) integration, self-determination acts as a conductor of the systemic meaning (for example, a purpose of functioning, or an idea of the sociocultural system, or an incentive motive, etc.), which determines the uniformity of connections of all interacting elements of the system. In this case, any element integrated into the sociocultural system, becomes the joint owner of the property of self-determination in this particular system. It is its interaction and interconnection with other elements that provides the structural and functional unity, leading to achievement of the purpose of functioning, etc. In turn, the exclusion of one of the elements will affect the structure and target functioning of the system, and, thereby, will cause deterioration in self-determination in the sociocultural system. It follows that systems possessing the semantic unity of their elements and the causal unity of the means of expression and dissemination of this meaning can be integrated into social reality. It is in such systems that semantic integration (disintegration) has priority in the formation (destruction) of self-determination. Thus, the descriptor of self-determination can be and should be presented at the meta-theoretical level of the sociocultural system description, i.e. at the level of the rules for constructing the selfdetermination theory.

Self-determination, in this case, manifests itself as a system property of the activity of the sociocultural system as a whole.

It follows from this statement that any sociocultural system determines its goals, direction of their achievement, mechanisms, structures of change, i.e. has the ability to selfdetermination. This property is manifested in the ability to independently form reactions to external conditions or determinants. External conditions cannot change the essential characteristics, target system settings. In particular, Pitirim Sorokin points out: "External conditions affect the development of the system, but mainly as a factor slowing down or accelerating the disclosure of its internal purpose ... however, these circumstances cannot force the system to manifest what it does not have; to become what it cannot become for internal reasons; to do what it is not able to do" $[9,10]$. Thus, self-determination underlies the preservation by the system of its own identity and the implementation of selfmanagement of its own development.

What determines the internal potential of self-determination, which allows it to successfully resist external influences or, in the absence of external controls, carry out development in the target direction? We find the answer in the following regularity revealed by Pitirim Sorokin: "All things being equal (including external), selfdetermination in determining its own destiny (as well as autonomy from the environment) is stronger, the stronger and better the degree of their integration is" $[9,10]$.

The level of self-determination of the sociocultural system and the potential for development in the target direction are directly proportional to the degree of semantic and causal integration of its elements.

This postulate determines the need to use methods and tools of system analysis that correspond to the system level of problem solving. First of all, we note the need for a transition to cognitive analysis based on a model description of the cause-and-effect relationships of the system. From the point of view of cognitive analytics, a model is a system of structurally functional description of the self-determination process, where the cause-and-effect relationships between the model factors reflect the causality of integration, and the logical-semantic connection is expressed in the target organization [10]. So, the cognitive model represents analytics, the situation studied in the sociocultural system, in the 
form of a visual graphic image. The elements subject to logical-semantic integration are factors (internal determinants), including the target factor, and many arcs (edges) of the graph reflect the relationship between factors. In social analytics, the use of named points of a graph (factors of a cognitive model) gives a researcher such a powerful mechanism as semantics of a situation. Based on its understanding, the analyst gets the opportunity to assess the logical-semantic correspondence of connections to the goals of selfdetermination. Moreover, already at this stage of the description of self-determination as a descriptor, there is a possibility of a preliminary assessment of the multiplicity of such relations. To formulate a description of causal integration, a transition to the use of oriented signed graphs will be required, i.e. graphs the arcs of which have a direction and note the influence of this connection [11]. In this case, arcs (otherwise, edges of the graph) represent cause-and-effect relationships, i.e. form a description of the causal model, which actually can be used as a descriptor of self-determination.

Thus, the set of factors united by cause-and-effect relationships in an oriented named signed graph reflects both the integration causation and the logical-semantic target organization of the sociocultural system. This is how self-determination is described as the main reason for the sociocultural system to set its own identity and manage its own development, i.e. its descriptor. So, on the basis of the figurative approximation of the essence of the studied system property of self-determination and its visual metaphorical representation in the form of a cognitive model, we get the opportunity to study "the main semantic content in terms of a certain field of knowledge", i.e. self-determination descriptor.

In a cognitive analysis of the system's self-determination by assessing the degree of target integration, it is necessary to identify all cause-and-effect relationships that provide a logical-semantic focus on the desired (target) result.

It should be noted that in this case social analysts rely on the corresponding repertoire of cognitive models, which allows one to quite strictly isolate from the set of system connections only their target-oriented logical-semantic and causal aggregate in the form of mental images and schemes. These schemes will allow the researcher to further evaluate the causal integration of the elements of the system or process under consideration, and in the future not only determine the level of self-determination of the system, but also predict the achievement of goals on the basis of assessing the potential for development.

The use of the self-determination descriptor in the form of a cognitive model and analysis of the degree of casual integration of the model target schemes open the way to effective cognitive modelling, construction of dynamic models, application of game and scenario analysis methods. At the same time, the analysis of the causal integration of the elements of the sociocultural system or process using cognitive models makes it quite easy to determine the level of system self-determination not only at a qualitative but also at a quantitative level.

\section{Conclusion}

Within the framework of the sociocultural system, self-determination acts as a conductor of a systemic meaning that determines the uniformity of connections of all interacting elements. Self-determination is manifested as a system property of the sociocultural system activity as a whole. Moreover, the level of self-determination of the sociocultural system and the potential for development in the target direction are directly proportional to the degree of semantic and causal integration of its elements.

Based on the figurative approximation of the essence of the studied system property of self-determination and its visual metaphorical representation in the form of a cognitive model, we get the opportunity to study the descriptor of self-determination. At the same 
time, the set of factors combined by cause-and-effect relationships in an oriented named signed graph reflects both the integration causation and the logical-semantic target organization of the sociocultural system.

Analysis of the causal integration of the elements of the sociocultural system or process using cognitive models makes it quite simple to determine the level of self-determination of a system not only at a qualitative but also at a quantitative level.

The article was prepared as part of the implementation of the internal grant of the Southern Federal University (VnGr-07/2017-20)

\section{References}

1. E.L. Deci, R.M. Ryan, Self-Determination Research: Reflections and Future Directions (Handbook of Self-Determination Research. Rochester, NY: University of Rochester Press, 2002).

2 R.M. Ryan, E.L. Deci, Self-Determination Theory and the Facilitation of Intrinsic Motivation, Social Development and Well-Being, American Psychologist, 55 (1), 74 (2000).

3. O.E. Dergacheva, Autonomy and Self-Determination in the Psychology of Motivation: the Theory of E. Deci and R. Ryan ('ed. by D.A. Leontiev') (Modern Psychology of Motivation, 2002).

4. N.A. Stepanova, The Development Model of Self-Determination as the Basis for Prevention of Addictive Behaviour of Adolescents in Modern Educational Organizations, Psychological and Pedagogical Research, 9 (4), 21-32 (2017). DOI:10.17759/psyedu.2017090403

5. I.Y. Labzova, The Theory of Self-Determination and Its Application in International Educational Practice, Human and Education, 3 (52), 152-156 (2017).

6. E.Y. Mandrikova, The Theory of Self-Determination in an Organizational Context: a Review of Foreign Research, Psychology. Journal of the Higher School of Economics, 7 (4), 120-140 (2010).

7. V.V. Makarov, Self-Determination of Crime through Its Organized Forms, Volga Pedagogical Search, 4 (6), 142-144 (2013).

8. E.V. Bochkareva, To the Issue of Forms of Self-Determination of Crime, Bulletin of Chelyabinsk State University, Series: Law, 2 (4), 70-71 (2017).

9. P. Sorokin, Social and Cultural Dynamics: a Study of Changes in Large Systems of Art, Truth, Ethics, Law and Public Relations (translated from English, commented and article by V.V. Sapov. Saint Petersburg, Russian Christian Academy of Humanities, 1056, 2000).

10. V.P. Durin, V.A. Semenov, Conflict as a Social Contradiction (Publishing House of Far Eastern State Transport University, Khabarovsk, 2008).

11. V.N. Gurba, S.A. Dyuzhikov, M.D. Rozin, V.N. Ryabtsev, V.P. Svechkarev, Cognitive Approaches to the Generalized Analysis of Geopolitical Conflicts, International Journal of Pure and Applied Mathematics, 119 (17) 1459-1463 (2018). 